\title{
On the core of routing games with revenues
}

\author{
Arantza Estévez-Fernández • Peter Borm • \\ Marc Meertens • Hans Reijnierse
}

\begin{abstract}
Traveling salesman problems with revenues form a generalization of traveling salesman problems. Here, next to travel costs an explicit revenue is generated by visiting a city. We analyze routing problems with revenues, where a predetermined route on all cities determines the tours along subgroups. Corresponding routing games with revenues are analyzed. It is shown that these games have a nonempty core and a complete description of the core is provided.
\end{abstract}

Keywords Routing problems · Revenues · Core

\section{JEL Classification C71}

The authors thank two referees for their valuable suggestions for improvement. Special thanks go to Herbert Hamers for his helpful discussion and comments.

\footnotetext{
A. Estévez-Fernández $(\varangle)$

Department of Econometrics and OR, VU University Amsterdam, De Boelelaan 1105, 1081 HV Amsterdam, The Netherlands e-mail: mestevez@feweb.vu.nl

P. Borm · H. Reijnierse

Center and Department of Econometrics and OR, Tilburg University, P.O. Box 90153, 5000 LE Tilburg, The Netherlands

M. Meertens

General Insurance Actuarial Risk Management (GARM), Reaal Verzekeringen, Croeselaan 1 B03.24, P.O. Box 8444, 3503 RK Utrecht, The Netherlands
} 


\section{Introduction}

In a traveling salesman (TS) situation a salesman, starting in his home city, has to visit a set of cities exactly once and has to come back to his home city at the end of the journey. Associating travel costs to connections the problem is how to find a tour with minimal cost. It is known that TS problems are NP-hard in general. For a survey on TS problems we refer to Lawler et al. (1997).

Fishburn and Pollak (1983) introduced the cost allocation problem that arises when each city (except the home city) corresponds to a player. The cost allocation is concerned with a fair allocation of the joint costs of the cheapest tour. This cost allocation problem was first studied within the framework of game theory by Potters et al. (1992) by introducing TS games. In a TS game, the value of a coalition of cities is the value of the cheapest tour in the TS problem associated to the coalition. Here, only (and exactly) the cities in the coalition will be visited. If the triangular inequalities are satisfied every 3-, 4-, and 5-person TS game has a nonempty core (Potters et al. 1992; Tamir 1989; Kuipers 1993). In the same setting however Tamir (1989) provides an example of a 6-person TS game with an empty core. In Okamoto (2004) it is shown that if the associated cost matrix is symmetric and has the Monge property, then the corresponding TS game is totally balanced (i.e. every subgame has a nonempty core). In Estévez-Fernández et al. (2006) it is seen that these results can be generalized to multiple (longest) traveling salesman (M(L)TS) games. In an MTS problem the salesman has to visit each city exactly once except for the home city which can be revisited as many times as desired. In a longest traveling salesman (LTS) problem there are profits associated to connections instead of travel costs. Hence, the objective of an LTS problem is to find a tour with maximal profit.

In Potters et al. (1992) also the class of fixed routing games is introduced. Here, the route along all cities is predetermined (e.g. by restrictions in the agenda of the salesman) and this tour determines the tours along all possible coalitions. Since the chosen tour and its associated cost are provided by the salesman, coalitions do not have any control on the tour that the salesman follows to visit them. Hence, the value of a coalition of a routing game is defined as the cost associated to the tour that visits the members of the coalition in the same relative order as in the predetermined tour. Potters et al. (1992) show that routing games have a nonempty core if the predetermined tour is an optimal tour for the related TS problem. Derks and Kuipers (1997) give a time efficient algorithm to provide core elements of a routing game. For a more extensive motivation of the model of routing games we refer to Derks and Kuipers (1997).

This paper studies routing problems with revenues: next to travel costs and a predetermined route on all cities, revenues of a visit are explicitly modeled and taken into account. Note that since the revenues obtained by the visit of a salesman are explicitly given, it might be the case that some of the cities will not be visited by the salesman if the objective is to maximize total joint profit. We will assume that the predetermined route is optimal in this sense and indeed visits all cities. Still, it might be optimal for a coalition not to visit all its cities in the prescribed relative order. Hence, the value of the associated routing game for a specific coalition is defined as the maximum attainable profit by one of its subcoalitions if the salesman visits all cities in this subcoalition 
in the relative order given by the predetermined route on all cities. We will show that every routing game with revenues has a nonempty core. Moreover, a complete description of the core is provided.

The idea of analyzing cost problems arising from a general service facility by taking explicitly into account the profits that the service will generate is not new. It was first studied in Littlechild and Owen (1976) within the framework of airport problems, with a more recent follow up in Brânzei et al. (2006). Okamoto (2004) briefly discusses prize-collecting traveling salesman problems where a reward is associated to each city and it is obtained only if the salesman visits the city. Meertens and Potters (2006) consider fixed tree games with revenues. Suijs et al. (2005) study the sharing of costs and revenues within a public network communication structure.

\section{Routing games with revenues}

Let $N=\{1,2, \ldots, n\}$ denote the set of cities that a traveling salesman has to visit. Let $N_{0}:=N \cup\{0\}$ where 0 denotes the traveling salesman's home city. Let $C=\left(c_{i j}\right)$ be an $N_{0} \times N_{0}$-matrix where $c_{i j}$ represents the costs to go from city $i$ to city $j$. Throughout this article we assume that:

(i) $c_{i i}=0$ for all $i \in N_{0}$,

(ii) $c_{i j}=c_{j i} \quad$ for all $i, j \in N_{0}, \quad$ (symmetry)

(iii) $c_{i j} \leq c_{i 0}+c_{0 j}$ for all $i, j \in N$. (triangle inequalities)

Note that the triangular inequalities are only required with respect to the home city to ensure willingness to cooperate among players: going back to the home city is always at least as expensive as going directly from city $i$ to city $j$. Potters et al. (1992) already pointed out that only this type of triangular inequalities are important for the analysis of nonemptiness of the core of routing games.

Whenever city $i \in N$ is visited by the traveling salesman, a revenue $b_{i} \geq 0$ is obtained. Due to the explicit modeling of the revenues we assume that the salesman, who starts from city 0 , visits each city at most once, and only returns to city 0 at the end of the journey.

Let $R \subset N$ and set $R_{0}:=R \cup\{0\}$. A bijection $\pi: R_{0} \rightarrow R_{0}$ is called a cyclic permutation if $\min \left\{t \in \mathbb{N} \mid \pi^{t}(i)=i\right\}=|R|+1$ for every $i \in R_{0}$. We denote by $\Pi(R)$ the set of all cyclic permutations on $R_{0}$. A cyclic permutation $\pi$ corresponds to a tour along $R$ : it starts in 0 and visits each city in $R$ exactly once returning to 0 at the end of the trip. Here, $\pi(i)$ is the city immediately visited after city $i$ for all $i \in R_{0}$. For convenience, we sometimes denote city 0 also by $n+1$ and in particular $\pi(i)=n+1$ means that $i$ is the last city on the tour. For $\pi \in \Pi(R)$, we denote by $c(\pi, R)$ the cost associated to the tour induced by $\pi$, i.e. $c(\pi, R)=\sum_{i \in R_{0}} c_{i \pi(i)}$. Consequently, the minimal cost $c(R)$ of a tour along $R$ is given by

$$
c(R):=\min _{\pi \in \Pi(R)}\{c(\pi, R)\}
$$


The total profit $\mathrm{p}(R)$ obtained when the salesman has visited all cities in $R$ according to a tour with minimal cost is

$$
\mathrm{p}(R):=b(R)-c(R)
$$

where $b(R):=\sum_{i \in R} b_{i}$.

Due to the revenue structure it may be more profitable for $N$ not to make a (complete) tour on $N$ itself but on a subset $R \subset N$, leaving $N \backslash R$ unvisited. Therefore, the optimization problem for $N$ boils down to finding a subset of cities $R$ such that $\mathrm{p}(R)$ is maximal. We denote the maximal profit for $N$ by $v(N)$, i.e.

$$
v(N):=\max _{R \subset N}\{\mathrm{p}(R)\}
$$

From now on we assume that it is optimal to visit all cities in $N$ via the cyclic permutation $\hat{\pi} \in \Pi(N)$. We also assume without loss of generality that

the optimal order $\hat{\pi}$ for $N$ is given by $0-1-2-\cdots-n-0$.

Hence, $v(N)=\mathrm{p}(N)$.

Associating each city in $N$ with a player, the question we would like to address is how to share $v(N)$ among the players. For this we choose the "routing" approach, where $\hat{\pi}$ determines the order in which potential subcoalitions are visited.

For $S \subset N$, the cyclic permutation $\hat{\pi}_{S} \in \Pi(S)$ induced by $\hat{\pi}$ is obtained from $\hat{\pi}$ by skipping the cities in $N \backslash S$ and leaving the order of the remaining cities unchanged. Formally, $\hat{\pi}_{S}$ is given by

$$
\hat{\pi}_{S}(i)=\hat{\pi}^{t(i)}(i) \text { for every } i \in S_{0}
$$

where $t(i):=\min \left\{t \in \mathbb{N} \mid \hat{\pi}^{t}(i) \in S_{0}\right\}$. With a minor abuse of notation we denote $c\left(\hat{\pi}_{S}, S\right)$ by $c(\hat{\pi}, S)$.

A coalition $S \subset N$ need not decide on the complete tour $\hat{\pi}_{S}$ on $S$ : a tour $\hat{\pi}_{R}$ on a subset $R \subset S$ may be more profitable. Hence, we define the value $v_{\hat{\pi}}(S)$ in the routing game $\left(N, v_{\hat{\pi}}\right)$ by

$$
v_{\hat{\pi}}(S)=\max _{R \subset S}\{b(R)-c(\hat{\pi}, R)\}
$$

Note that $v_{\hat{\pi}}(N)=v(N)$.

Example 2.1 Consider the routing problem with revenues represented in Fig. 1 where the numbers at the edges represent the travel costs and the boldface numbers at the nodes represent the revenues.

Note that assumption (2.1) is satisfied. The associated routing game with revenues has values: $v_{\hat{\pi}}(\{1\})=3, v_{\hat{\pi}}(\{2\})=0, v_{\hat{\pi}}(\{3\})=3, v_{\hat{\pi}}(\{1,2\})=5, v_{\hat{\pi}}(\{1,3\})=7$, $v_{\hat{\pi}}(\{2,3\})=3$ and $v_{\hat{\pi}}(N)=9$. 
Fig. 1 The routing problem with revenues in Example 2.1

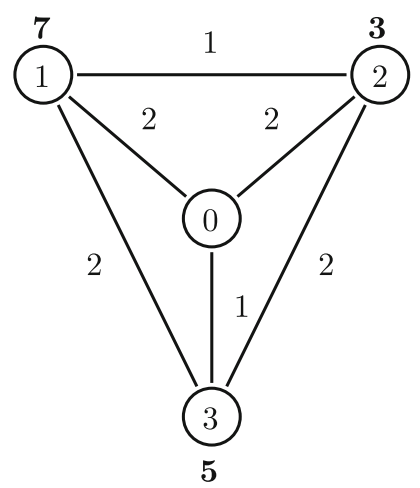

Note that if the revenues are so high that the salesman visits all cities of every coalition, then the routing game with revenues is strategically equivalent ${ }^{1}$ to a routing game à la Potters et al. (1992) and Derks and Kuipers (1997).

\section{The core}

In this section we study the core of routing games with revenues. We show that routing games with revenues have a nonempty core. Moreover, we give an intuitive interpretation of all core elements.

Let $(N, v)$ be a cooperative game. Recall that the core of $(N, v)$ is given by

$$
\operatorname{Core}(v)=\left\{x \in \mathbb{R}^{N} \mid x(N)=v(N), x(S) \geq v(S) \text { for all } S \in 2^{N}\right\}
$$

i.e. the core is the set of efficient allocations of $v(N)$ such that there is no coalition with an incentive to split off.

In the following example we illustrate that taking into account revenues has a definite impact on the structure of the core.

Example 3.1 Consider the routing problem with revenues represented in Fig. 2.

It is readily checked that assumption (2.1) is satisfied and that $v_{\hat{\pi}}(S)=0$ for every $S \subset N, S \neq N$, and $v_{\hat{\pi}}(N)=2$. Therefore,

$$
\operatorname{Core}\left(v_{\hat{\pi}}\right)=\operatorname{conv}\{(2,0,0),(0,2,0),(0,0,2)\}
$$

Consider now the associated (cost) routing game à la Potters et al. (1992) and Derks and Kuipers (1997) in which the revenues are explicitly not considered: they are high enough. One readily verifies that $c_{\hat{\pi}}(\{1\})=2, c_{\hat{\pi}}(\{2\})=6, c_{\hat{\pi}}(\{3\})=6$,

\footnotetext{
1 Here, we make a slight abuse of language when we say that a routing game with revenues is strategically equivalent to a (cost) routing game. We mean that there exist $k \in \mathbb{R}_{++}, a \in \mathbb{R}^{N}$ and $(N, c)$, a (cost) routing game, such that $v_{\hat{\pi}}(S)=a(S)-k c(S)$ for every $S \subset N$.
} 
Fig. 2 The routing problem with revenues in Example 3.1

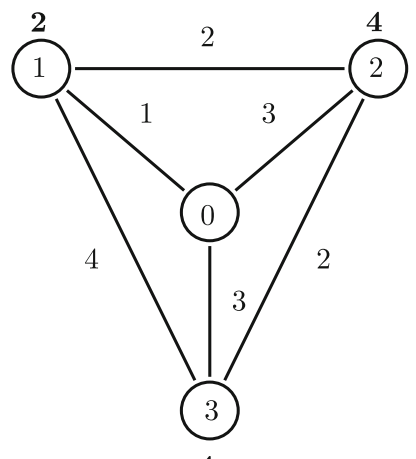

4

$c_{\hat{\pi}}(\{1,2\})=6, c_{\hat{\pi}}(\{1,3\})=8, c_{\hat{\pi}}(\{2,3\})=8$ and $c(N)=8$. Here $^{2}$

$$
\operatorname{Core}\left(c_{\hat{\pi}}\right)=\operatorname{conv}\{(2,0,6),(0,2,6),(0,6,2),(2,4,2)\} .
$$

Hence, there is not an obvious relation between $\operatorname{Core}\left(c_{\hat{\pi}}\right)$ and $\operatorname{Core}\left(v_{\hat{\pi}}\right)$. Moreover, it is readily checked that the above routing game with revenues is not strategically equivalent to any routing game à la Potters et al. (1992) and Derks and Kuipers (1997).

Next, we show that a routing game with revenues, $\left(N, v_{\hat{\pi}}\right)$, corresponding to travel cost matrix $C \in \mathbb{R}^{N_{0} \times N_{0}}$ and revenue vector $b \in \mathbb{R}^{N}$ has a nonempty core.

For every $S \subset N$ we define the linear programming problem $\operatorname{LP}(S)$ by:

$$
\begin{aligned}
\operatorname{maximize} & \sum_{i=0}^{n} \sum_{j=i+1}^{n+1}\left(b_{j}-c_{i j}\right) x_{i j} \\
\text { s.t. } & \sum_{k=0}^{i-1} x_{k i} \leq e_{i}^{S} \quad \text { for all } i \in\{1, \ldots, n\} ; \\
& \sum_{k=0}^{i-1} x_{k i}-\sum_{j=i+1}^{n+1} x_{i j}=0 \quad \text { for all } i \in\{1, \ldots, n\} ; \\
& x_{i j} \geq 0 \quad \text { for all } i, j \in\{0,1, \ldots, n, n+1\} \text { with } i<j .
\end{aligned}
$$

with $b_{0}=b_{n+1}:=0$ and $c_{i n+1}:=c_{i 0}$ and where $e^{S} \in \mathbb{R}^{N}$ is a vector of zeros and ones with $e_{i}^{S}=1$ if $i \in S$ and $e_{i}^{S}=0$ otherwise.

It is readily checked that $\operatorname{LP}(S)$ is feasible and bounded. Here, $x_{i j}$ can be interpreted as the "amount of flow that goes from $i$ to $j$ ". The profit obtained per unit of flow from $i$ to $j$ is $b_{j}-c_{i j}$ for every $i$ and $j$ such that $0 \leq i<j \leq n+1$ and the objective

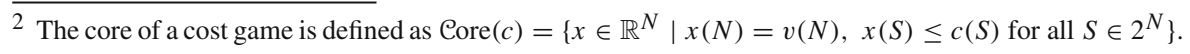


function is to maximize the total profit as represented in (3.1). Equation (3.2) indicates that the total flow "arriving" at city $i$ can not exceed one unit of flow, i.e. one can think of this as a capacity restriction on the nodes. Equation (3.3) makes sure that the amount of flow "arriving" at $i$ equals the amount of flow "leaving" $i$.

Note that the game $(N, u)$ with $u(S)$ defined as the optimal value of $\operatorname{LP}(S)$ for $S \subset N$, is a linear production game and therefore it has a nonempty core (Owen 1975).

Lemma $3.1 \quad$ (i) $u(S) \geq v_{\hat{\pi}}(S)$ for every $S \subset N$.

(ii) $u(N)=v_{\hat{\pi}}(N)$.

Proof (i) Let $S \subset N$ and $R \subset S$ such that

$$
v_{\hat{\pi}}(S)=b(R)-c(\hat{\pi}, R)
$$

If $R=\emptyset$, then $v_{\hat{\pi}}(S)=0 \leq u(S)$. Otherwise, $R:=\left\{i_{1}, \ldots, i_{r}\right\}$ with $i_{1}<\cdots<i_{r}$. Define

$$
x_{i j}:=\left\{\begin{array}{ll}
1 & \text { if } i \in\left\{0, i_{1}, \ldots, i_{r-1}\right\} \\
0 & \text { otherwise. }
\end{array} \text { and } j=\hat{\pi}_{R}(i), \text { or } i=i_{r} \text { and } j=n+1,\right.
$$

Then, $\left(x_{i j}\right)_{0 \leq i<j \leq n+1}$ is a feasible solution of $\operatorname{LP}(S)$ and

$$
\begin{aligned}
u(S) & \geq \sum_{i=0}^{n} \sum_{j=i+1}^{n+1}\left(b_{j}-c_{i j}\right) x_{i j} \\
& =b_{i_{1}}+\cdots+b_{i_{r}}-\left(c_{0 i_{1}}+c_{i_{1} i_{2}}+\cdots+c_{i_{r-1} i_{r}}+c_{0 i_{r}}\right) \\
& =v_{\hat{\pi}}(S) .
\end{aligned}
$$

(ii) By (i) it suffices to show that $u(N) \leq v_{\hat{\pi}}(N)$. Note that $\mathrm{LP}(N)$ is a transportation problem with $\{0,1, \ldots, n\}$ the set of sources, $\{1, \ldots, n, n+1\}$ the set of sinks, and such that there are no links going from a source $i$ to a sink $j$ with $i>j$ and the reward when going from $i \in N$ to itself is zero. Then, there exists an integral optimal solution, $\bar{x}=\left(\bar{x}_{i j}\right)_{0 \leq i<j \leq n+1}$, for $\operatorname{LP}(N)$ (see Nemhauser and Wolsey 1988, Chap. I.3, Corollary 5.2). Moreover,

(i) $\bar{x}_{i j} \in\{0,1\}$ for every $i, j$ with $0 \leq i<j \leq n+1$ by Eqs. (3.2) and (3.4).

(ii) If $\sum_{k=0}^{i-1} \bar{x}_{k i}=1$, then there exists a unique $k(i) \in\{0, \ldots, i-1\}$ such that $\bar{x}_{k(i) i}=1$ by (i).

(iii) If $\sum_{k=0}^{i-1} \bar{x}_{k i}=1$, then there exists a unique $j(i) \in\{i+1, \ldots, n+1\}$ such that $\bar{x}_{i j(i)}=1$ by Eq. (3.3).

(iv) $\sum_{i=1}^{n} \bar{x}_{0 i}=\sum_{i=1}^{n} \bar{x}_{i n+1}$ by Eq. (3.3).

Let $N(\bar{x}):=\left\{i \in N \mid \sum_{k=0}^{i-1} \bar{x}_{k i}=1\right\}$ and let $\left\{i_{1}, \ldots, i_{r}\right\}=\left\{i \in N(\bar{x}) \mid \bar{x}_{0 i}=1\right\}$. Let $t\left(i_{l}\right)$ be the smallest integer such that $j^{t\left(i_{l}\right)}\left(i_{l}\right)=n+1$. We define

$$
N_{l}(\bar{x})=\left\{j^{t}\left(i_{l}\right) \mid t \in\left\{1, \ldots, t\left(i_{l}\right)-1\right\}\right.
$$


for $l \in\{1, \ldots, r\}$. It is readily checked that $N_{1}(\bar{x}), \ldots, N_{r}(\bar{x})$ is a partition of $N(\bar{x})$. Moreover, note that this partition implies that $r$ tours, $\pi^{1} \in \Pi\left(N_{1}(\bar{x})\right), \ldots, \pi^{r} \in$ $\Pi\left(N_{r}(\bar{x})\right)$, are followed in the optimal solution, where tour $\pi^{l}$ is given by $0-i_{l}-$ $\cdots-j^{t\left(i_{l}\right)-1}\left(i_{l}\right)-(n+1)$ for $l \in\{1, \ldots, r\}$ (See Fig. 3).

Define $\tilde{\pi} \in \Pi(N(\bar{x}))$ by $0-i_{1}-\cdots-j^{t\left(i_{1}\right)-1}\left(i_{1}\right)-i_{2}-\cdots-j^{t\left(i_{2}\right)-1}\left(i_{2}\right)-\cdots-$ $i_{r}-\cdots-j^{t\left(i_{r}\right)-1}\left(i_{r}\right)-(n+1)$ (See Fig. 4).

Hence,

$$
\begin{aligned}
u(N) & =\sum_{i=0}^{n+1} \sum_{j=i+1}^{n+1}\left(b_{j}-c_{i j}\right) \bar{x}_{i j} \\
& =\sum_{l=1}^{r} \sum_{i \in N_{l}(\bar{x}) \cup\{0\}}\left(b_{\pi^{l}(i)}-c_{i \pi^{l}(i)}\right) \\
& =\sum_{l=1}^{r} b\left(N_{l}(\bar{x})\right)-\sum_{l=1}^{r} c\left(\pi^{l}, N_{l}(\bar{x})\right) \\
& \leq b\left(\cup_{l=1}^{r} N_{l}(\bar{x})\right)-c\left(\tilde{\pi}, \cup_{l=1}^{r} N_{l}(\bar{x})\right) \\
& \leq b\left(\cup_{l=1}^{r} N_{l}(\bar{x})\right)-c\left(\cup_{l=1}^{r} N_{l}(\bar{x})\right) \\
& \leq b(N)-c(N)=v_{\hat{\pi}}(N)
\end{aligned}
$$

where the first inequality holds by the triangular inequalities, the second one holds by definition of $c\left(\cup_{l=1}^{r} N_{l}(\bar{x})\right)$ and the last one by assumption (2.1) and because it is optimal to visit all cities in $N$ by assumption.

Note that if two games $(N, v)$ and $(N, u)$ are such that $v(S) \leq u(S)$ for every $S \subset N, v(N)=u(N)$, and $\operatorname{Core}(u) \neq \emptyset$, then $\operatorname{Core}(v) \neq \emptyset$ and $\operatorname{Core}(u) \subset \operatorname{Core}(v)$. Hence, as a direct consequence of Lemma 3.1 we have that a routing game with revenues has a nonempty core.

Theorem 3.2 Any routing game with revenues has a nonempty core.

The following result gives a full description of the core of a routing game with revenues. ${ }^{3}$ It states that an allocation $x$ belongs to the core of the game if each coordinate $x_{i}$ can be written as $x_{i}=b_{i}-c_{i-1 i}+z_{i-1}-z_{i}$. This can be interpreted in the following way: First of all, player $i$ obtains the revenue $b_{i}$ when the salesman visits its city and has to pay the travel costs $c_{i-1 i}$ from city $i-1$ to city $i$. Next, since player $i-1$ also gets revenues from the visit, it helps player $i$ with the travel costs by paying a compensation $z_{i-1}$. In a similar way, player $i$ helps player $i+1$ with the travel costs of the trip from city $i$ to city $i+1$ with $z_{i}$. Equation (3.6) below reflects that player $i$ never compensates $i+1$ more than the total amount he gets once $i-1$ has paid the compensation. Equation (3.7) reflects the fact that player $j+1$ indeed prefers that the salesman comes from player $j$ instead of another player $i(<j)$.

\footnotetext{
${ }^{3}$ Since routing games with revenues generalize routing games, this description of the core can also be applied to routing games.
} 
Fig. 3 Representation of the tours given by $\pi^{l}$

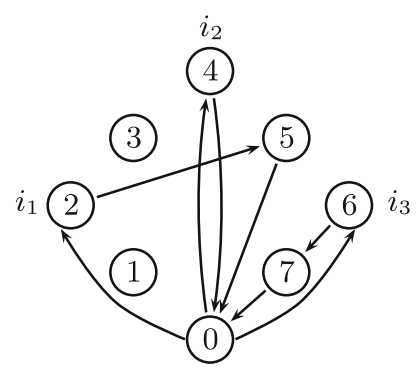

Fig. 4 Representation of $\tilde{\pi}$

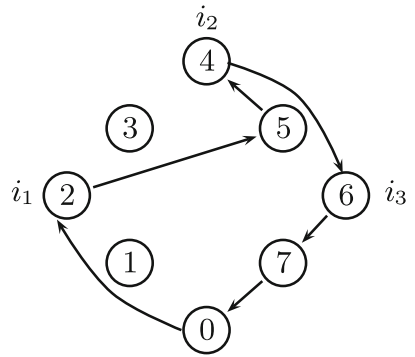

Theorem 3.3 Let $\left(N, v_{\hat{\pi}}\right)$ be a routing game with revenues corresponding to travel cost matrix $C \in \mathbb{R}^{N_{0} \times N_{0}}$ and revenue vector $b \in \mathbb{R}^{N}$. Then, the following three assertions are equivalent.

(i) $x \in \operatorname{Core}\left(v_{\hat{\pi}}\right)$.

(ii) $x \geq 0, x(N)=v_{\hat{\pi}}(N)$, and $x(N \backslash S) \geq v_{\hat{\pi}}(N \backslash S)$ for every $S \subset N$ with $S=\{i, i+1, \ldots, j\}$ and $i \leq j$.

(iii) $x_{i}:=b_{i}-c_{i-1 i}+z_{i-1}-z_{i}$ for all $i \in\{1, \ldots, n\}$ with

$$
\begin{gathered}
z_{0}:=0, z_{n}:=c_{0 n} \\
z_{i}-z_{i-1} \leq b_{i}-c_{i-1 i} \text { for all } i \in\{1, \ldots, n\} \\
z_{j}-z_{i} \geq c_{j j+1}-c_{i j+1} \text { for all } i, j \in\{0,1, \ldots, n\} \text { with } i<j .
\end{gathered}
$$

Proof (i) $\Rightarrow$ (ii) is immediate and therefore omitted.

(ii) $\Rightarrow$ (iii) Let $x \in \mathbb{R}^{N}$ satisfy the conditions mentioned in assertion (ii) of the theorem. Define the vector $z \in \mathbb{R}^{N_{0}}$ as follows:

$$
\begin{gathered}
z_{0}:=0 \\
z_{i}:=b_{i}-c_{i-1 i}+z_{i-1}-x_{i} \text { for every } i \in\{1, \ldots, n\} .
\end{gathered}
$$

It is readily checked that $z_{i}=\sum_{k=1}^{i} b_{k}-\sum_{k=1}^{i} c_{k-1 k}-\sum_{k=1}^{i} x_{k}$ for all $i \in$ $\{1, \ldots, n\}$. Hence, 


$$
\begin{aligned}
z_{n} & =b(N)-\sum_{k=1}^{n} c_{k-1 k}-x(N) \\
& =b(N)-\sum_{k=1}^{n} c_{k-1 k}-\left(b(N)-\sum_{k=1}^{n+1} c_{k-1 k}\right) \\
& =c_{n n+1}=c_{0 n}
\end{aligned}
$$

where the second equality follows from $x(N)=v_{\hat{\pi}}(N)$. Consequently, Eq. (3.5) holds.

With respect to Eq. (3.6), clearly $x \geq 0$ implies $z_{i}-z_{i-1} \leq b_{i}-c_{i-1 i}$.

Next, we show Eq. (3.7), i.e. $z_{j}-z_{i} \geq c_{j j+1}-c_{i j+1}$ for all $i, j \in\{0,1, \ldots, n\}$ with $i<j$. Suppose that there exist $i, j \in\{0,1, \ldots, n\}$ with $i<j$ be such that $z_{j}-z_{i}<c_{j j+1}-c_{i j+1}$. Define $S=\{i+1, \ldots, j\}$. Clearly, if we can show that $x(N \backslash S)<v_{\hat{\pi}}(N \backslash S)$ we arrive at a contradiction with one of the assumptions in (ii). Indeed,

$$
\begin{aligned}
x(N \backslash S)= & b(N \backslash S)-\left[c_{01}+\cdots+c_{i-1 i}+c_{j j+1}+\cdots+c_{n-1 n}\right] \\
& +\left[z_{0}+\cdots+z_{i-1}+z_{j}+\cdots+z_{n-1}\right] \\
& -\left[z_{1}+\cdots+z_{i}+z_{j+1}+\cdots+z_{n}\right] \\
= & b(N \backslash S)-\left[c_{01}+\cdots+c_{i-1 i}+c_{j j+1}+\cdots+c_{n-1 n}\right]+z_{j}-z_{i}-z_{n} \\
= & b(N \backslash S)-\left[c_{01}+\cdots+c_{i-1 i}+c_{j j+1}+\cdots+c_{n-1 n}+c_{n 0}\right]+z_{j}-z_{i} \\
< & b(N \backslash S)-\left[c_{01}+\cdots+c_{i-1 i}+c_{j j+1}+\cdots+c_{n-1 n}+c_{n 0}\right] \\
& +c_{j j+1}-c_{i j+1} \\
= & b(N \backslash S)-\left[c_{01}+\cdots+c_{i-1 i}+c_{i j+1}+\cdots+c_{n-1 n}+c_{0 n}\right] \\
= & b(N \backslash S)-c\left(\hat{\pi}_{N S S}, N \backslash S\right) \\
\leq & v_{\hat{\pi}}(N \backslash S),
\end{aligned}
$$

where the second equality follows from $z_{0}=0$, the third one is a consequence of $z_{n}=c_{0 n}$, the strict inequality follows from the assumption and the weak inequality is by definition of $v_{\hat{\pi}}$.

(iii) $\Rightarrow$ (i) Let $z \in \mathbb{R}^{N_{0}}$ satisfy the conditions (3.5), (3.6), and (3.7) mentioned in assertion (iii) of the theorem. Define $x_{i}:=b_{i}-c_{i-1 i}+z_{i-1}-z_{i}$ for all $1 \leq i \leq n$. It is readily checked that $x(N)=v_{\hat{\pi}}(N)$. Let $S \subset N$ be a coalition, and let $R:=\left\{i_{1}, \ldots, i_{r}\right\} \subset S$ be such that $v_{\hat{\pi}}(S)=b(R)-c\left(\hat{\pi}_{R}, R\right)$. It suffices to prove that $x(S) \geq v_{\hat{\pi}}(S)$. For this, note that

$$
\begin{aligned}
x(S) & \geq x(R)=b(R)-\sum_{k=1}^{r} c_{i_{k}-1 i_{k}}+\sum_{k=1}^{r} z_{i_{k}-1}-\sum_{k=1}^{r} z_{i_{k}} \\
& =b(R)-\sum_{k=1}^{r} c_{i_{k}-1 i_{k}}+\sum_{k=2}^{r} z_{i_{k}-1}-\sum_{k=1}^{r-1} z_{i_{k}}-z_{0}+z_{i_{1}-1}-z_{i_{r}}+z_{n}-c_{0 n}
\end{aligned}
$$




$$
\begin{aligned}
& \geq b(R)-\sum_{k=1}^{r} c_{i_{k}-1 i_{k}}+\sum_{k=2}^{r} z_{i_{k}-1}-\sum_{k=1}^{r-1} z_{i_{k}}+c_{i_{1}-1 i_{1}}-c_{0 i_{1}}-c_{0 i_{r}} \\
& =b(R)-\sum_{k=1}^{r} c_{i_{k}-1 i_{k}}+\sum_{k=1}^{r-1} z_{i_{k+1}-1}-\sum_{k=1}^{r-1} z_{i_{k}}+c_{i_{1}-1 i_{1}}-c_{0 i_{1}}-c_{0 i_{r}} \\
& =b(R)-\sum_{k=2}^{r} c_{i_{k}-1 i_{k}}+\sum_{k=1}^{r-1}\left[z_{i_{k+1}-1}-z_{i_{k}}\right]-c_{0 i_{1}}-c_{0 i_{r}} \\
& \geq b(R)-\sum_{k=2}^{r} c_{i_{k}-1 i_{k}}+\sum_{k=1}^{r-1}\left[c_{i_{k+1}-1 i_{k+1}}-c_{i_{k} i_{k+1}}\right]-c_{0 i_{1}}-c_{0 i_{r}} \\
& =b(R)-\sum_{k=1}^{r-1} c_{i_{k} i_{k+1}}-c_{0 i_{1}}-c_{0 i_{r}} \\
& =b(R)-c\left(\hat{\pi}_{R}, R\right) \\
& =v_{\hat{\pi}}(S) .
\end{aligned}
$$

Here, the first inequality is a consequence of Eq. (3.6) which implies $x_{i} \geq 0$ for every $i \in N$, the second inequality follows by applying Eq. (3.7) to $j=i_{1}-1, i=0$ and to $j=n, i=i_{r}$ and the second one is also an immediate consequence of Eq. (3.7).

Consider the vector $x \in \mathbb{R}^{N}$ defined recursively by

$$
x_{i}=v_{\hat{\pi}}(N)-\max _{k \leq i}\left\{v_{\hat{\pi}}(N \backslash\{k, \ldots, i\})+x(\{k, \ldots, i-1\})\right\}
$$

for $i \in\{1, \ldots, n\}$. This allocation can be interpreted as follows. Assume only connected coalitions (i.e. coalitions $\{k, k+1, \ldots, i\}$ ) are allowed to step out of the negotiations on the allocation of $v_{\hat{\pi}}(N)$ and stepping out is decided recursively by the individual players. Consider that player $i$ wants to step out. If the coalition $\{k, k+1, \ldots, i\}$ decides to step out, the players in $N \backslash\{k, k+1, \ldots, i\}$ further negotiate the allocation of $v_{\hat{\pi}}(N \backslash\{k, k+1, \ldots, i\})$ and each player $j \in\{k, k+1, \ldots, i-1\}$ already got $x_{j}$. Hence, player $i$ is left with $v_{\hat{\pi}}(N)-\left[v_{\hat{\pi}}(N \backslash\{k, \ldots, i\})+x(\{k, \ldots, i-\right.$ $1\})$ ]. Having no influence on "earlier" stepping out player $i$ can only claim the minimum compensation over the set of all possible connected coalitions $\{k, \ldots, i\}$ with $1 \leq k \leq i$ which is reflected in (3.8).

It turns out that the allocation $x$ defined by Eq. (3.8) is a core element of $v_{\hat{\pi}}$. This result is an immediate consequence of Theorem 4 in Derks and Kuipers (1997) and the description of the core by coalitions $N \backslash\{k, \ldots, i\}$ given in Theorem 3.3. Hence, the proof is omitted.

Theorem 3.4 Let $\left(N, v_{\hat{\pi}}\right)$ be a routing game with revenues corresponding to travel cost matrix $C \in \mathbb{R}^{N_{0} \times N_{0}}$ and revenue vector $b \in \mathbb{R}^{N}$. Let $x$ be defined as in Eq. (3.8). Then, $x \in \operatorname{Core}\left(v_{\hat{\pi}}\right)$. 


\section{Final remarks}

The dual $\left(N, c^{d}\right)$ of a TU-game $(N, c)$ is defined by

$$
c^{d}(S)=c(N)-c(N \backslash S) \text { for every } S \subset N .
$$

In Derks and Kuipers (1997) the core of routing games is analyzed by studying dual routing games. It turns out that dual routing games have the connectedness property (i.e. possibly after reindexing the players, all essential coalitions are of the form $\{i, \ldots, j\}$, with $i \leq j$, where a coalition $S$ is essential if $c^{d}(S)>\sum_{l=1}^{u} c^{d}\left(R_{l}\right)$ for every (non-trivial) partition $\left\langle R_{1}, \ldots, R_{u}>\right.$ of $S$ ). This together with the fact that routing games are $N$-subadditive (i.e. $c(N) \leq c(S)+c(N \backslash S$ ) for any $S \subset N$ ) suffices to show nonemptiness of the core for routing games. The following example shows that this type of result cannot be directly extended in general if one additionally considers revenues in the way of this paper. Additional information on the underlying cost structure that is not captured in the game is required.

Example 4.1 Consider the cost game $(N, c)$ described in Table 1 and let $b=(30,9$, $30)$. The corresponding dual game $\left(N, c^{d}\right)$ and cost-revenue game $(N, v)$ are also given in Table 1 . Here, following the ideas of this paper, $(N, v)$ is defined by

$$
v(S)=\max _{R \subset S}\{b(R)-c(R)\}
$$

It is readily checked that the dual game has the connectedness property and that $(N, c)$ is $N$-subadittive. Consequently, $c$ is balanced, but $(N, v)$ has an empty core since $v(\{1\})+v(\{2\})+v(\{3\})=20>19=v(N)$.

Note that $(N, c)$ corresponds to a routing game generated by the routing problem given in Fig. 5. This routing problem however violates the triangular inequalities since: $c_{13}=31>20=c_{10}+c_{03}$. Hence, the triangular inequalities are essential for our result on nonemptiness of the core.

We have analyzed the core of routing games with revenues in which the predetermined route is optimal for the associated combinatorial problem and visits all cities in $N$. Next, we provide an example that illustrates that our assumption (2.1) (i.e. the salesman visits all cities) is not restrictive. It turns out that if the salesman only visits some of the cities, those that are unvisited receive a payoff of zero in any core allocation and the various results provided in the previous sections are still valid.

Table 1 Coalitional values of the cost game, dual game, and

\begin{tabular}{lccccccc}
\hline$S$ & $\{1\}$ & $\{2\}$ & $\{3\}$ & $\{1,2\}$ & $\{1,3\}$ & $\{2,3\}$ & $\{1,2,3\}$ \\
\hline$c(S)$ & 20 & 20 & 20 & 35 & 51 & 35 & 50 \\
$c^{d}(S)$ & 15 & -1 & 15 & 30 & 30 & 30 & 50 \\
$v(S)$ & 10 & 0 & 10 & 10 & 10 & 10 & 19 \\
\hline
\end{tabular}
cost-revenue game in Example 4.1 
Fig. 5 The routing problem behind the cost game in Example 4.1

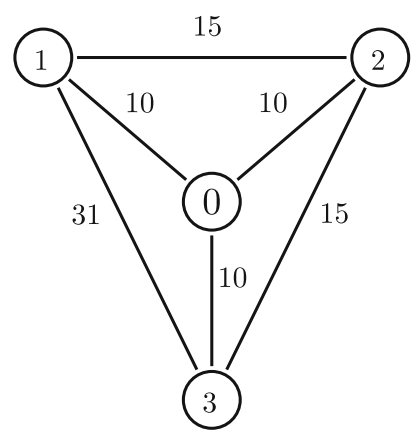

Fig. 6 The routing problem with revenues in Example 4.2

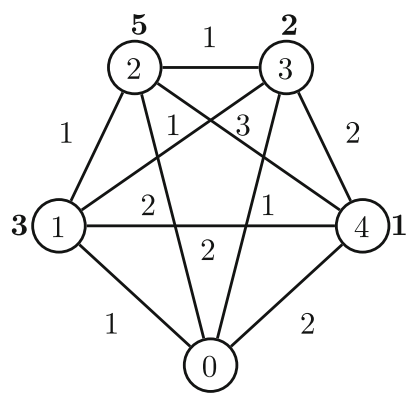

Example 4.2 Consider the routing problem with revenues represented in Fig. 6 where the numbers at the edges represent the traveling costs and the boldface numbers at the nodes represent the revenues.

It is readily seen that the optimal tour for this situation only visits the cities 1,2 , and 3 in the order $0-1-2-3-0$ denoted by $\hat{\pi}$. Hence the coalitional values of the routing game are: $v_{\hat{\pi}}(\{1\})=1, v_{\hat{\pi}}(\{2\})=1, v_{\hat{\pi}}(\{3\})=0, v_{\hat{\pi}}(\{4\})=0, v_{\hat{\pi}}(\{1,2\})=$ $4, v_{\hat{\pi}}(\{1,3\})=2, v_{\hat{\pi}}(\{1,4\})=1, v_{\hat{\pi}}(\{2,3\})=3, v_{\hat{\pi}}(\{2,4\})=1, v_{\hat{\pi}}(\{3,4\})=$ $0, v_{\hat{\pi}}(\{1,2,3\})=6, v_{\hat{\pi}}(\{1,2,4\})=4, v_{\hat{\pi}}(\{1,3,4\})=2, v_{\hat{\pi}}(\{2,3,4\})=3$ and $v_{\hat{\pi}}(N)=6$. Here, player 4 is a zero player and the core of the game is $\operatorname{Core}\left(v_{\hat{\pi}}\right)=$ $\operatorname{conv}\{(3,3,0,0),(3,1,2,0),(2,4,0,0),(1,4,1,0),(1,3,2,0)\}$. Note that the core can still be described by means of the cost of the tour, the vector of revenues, and a vector of compensations as in Theorem 3.3.

Open Access This article is distributed under the terms of the Creative Commons Attribution Noncommercial License which permits any noncommercial use, distribution, and reproduction in any medium, provided the original author(s) and source are credited.

\section{References}

Brânzei R, Iñarra E, Tijs S, Zarzuelo J (2006) A simple algorithm for the nucleolus of airport profit problems. Int J Game Theory 34:259-272

Derks J, Kuipers J (1997) On the core of routing games. Int J Game Theory 26:193-205

Estévez-Fernández A, Borm P, Hamers H (2006) On the core of multiple longest traveling salesman games. Eur J Oper Res 174:1816-1827 
Fishburn PC, Pollak HO (1983) Fixed-route cost allocation. Am Math Monthly 90:366-378

Kuipers J (1993) A note in the 5-person traveling salesman game. Zeitschrift für Operations Research 21:339-351

Lawler EL, Lenstra JK, Kan AHGR, Shmoys DB, Hurkens CAJ (1997) The traveling salesman problem: a guided tour of combinatorial optimization. Wiley-Interscience, Chichester

Littlechild SC, Owen G (1976) A further note on the nucleolus of the "airport game". Int J Game Theory 5:91-95

Meertens M, Potters J (2006) The nucleolus of trees with revenues. Math Methods Oper Res 64:363-382

Nemhauser GL, Wolsey LA (1988) Integer and combinatorial optimization. Wiley-Interscience, New York

Okamoto Y (2004) Traveling salesman games with the monge property. Discrete Appl Math 138:349-369

Owen G (1975) On the core of linear production games. Math Program 9:358-370

Potters JA, Curiel IJ, Tijs SH (1992) Traveling salesman games. Math Program 53:199-211

Suijs J, Borm P, Hamers H, Quant M, Koster M (2005) Communication and cooperation in public network situations. Ann Oper Res 137:117-140

Tamir A (1989) On the core of a traveling salesman cost allocation game. Oper Res Lett 8:31-34 